\title{
Opinion Analysis Using Domain Ontology for Implementing Natural Language Based Feedback System
}

\author{
Pratik K. Agrawal \\ Department of Computer Science, Prof Ram Meghe Institute of technology \& Research, Badnera, India \\ E-mail: pratik.agrawaal@gmail.com \\ Dr. Avinash. J. Agrawal \\ Department of Computer Science, Shri Ramdeobaba College of Engineering \& Management, Nagpur, India \\ E-mail: avinashjagrawal@gmail.com
}

\begin{abstract}
This paper proposes a natural language based feedback analysis system that extracts semantic relations from feedback data in order to map it with the domain ontology. After pre-processing a set of words or phrases are extracted from the input data. The data are analyzed semantically to interpret its meaning. This meaning is in an intermediate form which is then mapped to the terms defined in the ontology using similarity function. The opinion analysis of the semantic data is carried out for measuring the polarity of the feedback by the use of opinion analysis method. The system is evaluated on the input feedback data.
\end{abstract}

Index Terms - Natural Language Processing, Ontology, Semantic Analysis, Opinion Analysis

\section{Introduction}

Natural Language Processing is a theoretically motivated range of computational techniques for analyzing and representing naturally occurring texts at one or more levels of linguistic analysis for the purpose of achieving human-like language processing for a range of tasks or applications. There are different levels like semantic analysis, opinion analysis etc. That should be studied in order to understand this computational language.

In this semantic analysis means understanding the meaning of the words to which it represents. Semantic processing determines the possible meanings of a sentence by focusing on the interactions among wordlevel meanings in the sentence. Semantic disambiguation of words with multiple senses are included in this level of processing; in an analogous way to how syntactic disambiguation of words that can function as multiple parts-of-speech is accomplished at the syntactic level.
Opinion analysis means deciding the polarity of the words whether they are positive, negative and neutral and based on that calculating the total polarity of that entity due to this advantage natural language is used in the feedback analysis system. Now it is necessary to understand what feedback is and why it is essential?

Feedback is essential to the working and survival of all regulatory mechanisms found throughout living and non-living nature, and also in manmade systems such as education and economic system. Feedback is a two way flow as its inherent all interactions, between human-to-human, human-tomachine or machine-to-machine.

In the knowledge-based economy new ideas and models of thinking serve as a basis of organizational vitality. How to reveal student talents and make maximum use of them aimed at achieving the institute's main goal. The institute wants to see efficient processes and proof of student's personal growth. The opportunity is to increase intellectual capital via institute learning and efficient feedback.

Student's feedback is very essential for an educational institute success. It will tell and keep the organization updated about the organization's strengths and weaknesses. The institute will consider those weaknesses and try to eliminate it. It will help them in carrying Strength, Weaknesses, Opportunities and Threats analysis in an institute. It will help in contributing to the institute's growth.

Student feedback should be encouraged by the institute as it tells them how motivated and satisfied the Students are in an institute. It tells the institute the reactions of students to the institute's policies. The student should be honest enough in giving their views about a particular entity. They should be assured that their suggestions would be taken into consideration for implementation and shouldn't be hesitant in giving their feedback. 
Taking feedback is not enough the feedback should be properly evaluated for that feedback analysis system plays an important role. Feedback analysis means evaluating the feedback in an optimized way to implement suggestions given by the student in less time. Thus, the institute should encourage students to give feedback and take it into consideration for the growth and smooth running of the institute.

The remainder of this paper is organized as follows: Section 2 gives an effective motivation for the development of the feedback system. Section 3 describes the related work that helps in designing and implementing the system. Section 4 presents the proposed feedback system. Section 5 describes the result of the testing data that is carried out on the system. Conclusion and future work are given in the final section.

\subsection{Motivation}

The motivation of natural language based feedback system shall be described by an example. A person wants to provide the feedback for the organization. The organization will provide him with a feedback form. The online feedback from consists of a radio button. The radio button is marked with some specified values based on some calculation. The person wants to click on the given radio button for providing feedback to the organization.

Due to the use of a radio button, the person fails to express his feeling and also was not able to provide any suggestions regarding the organization. The organization is also not able to determine what the fault is in the system and so judgment regarding the problem cannot be made quickly.

So for all these problems, we can provide feedback systems that will analysis person day to day words. So that the person can easily express his views in his own words and can provide valuable suggestions for the organization. The organization can easily detect the problems by the person suggestions and judgment regarding that problems can be made easily and quickly. This will help us to save time and will helps in better development of the organization.

\section{Related work}

Opinion analysis has gained much attention from the research community in recent years. It is concerned with the problem of discovering emotional meanings in text, and most common tasks usually include emotion labeling, polarity recognition and subjectivity identification. The growing research interest is mainly due to the practical applications of opinion analysis. Companies and organizations are interested in finding out costumer opinions, while individuals are interested in others' opinions when purchasing a product or deciding whether or not watching a movie.
1. Jorge Carrillo de Albornoz, Laura Plaza, Pablo Gervás in their paper" A Hybrid Approach to Emotional Sentence Polarity and Intensity Classification" presents a new approach to sentence level opinion analysis. The aim was to determine whether a sentence expresses a positive, negative or neutral opinion, as well as its intensity. WSD is performed by the method over the words in the sentence in order to work with concepts, and makes use of the knowledge in an affective lexicon to label these concepts with emotional categories. It also deals with the effect of negations and quantifiers on polarity and intensity analysis. In two different domains extensive evaluation is performed in order to determine how the method behaves in 2-classes (positive and negative), 3-classes (positive, negative and neutral) and 5-classes (weakly positive, strongly positive, strongly negative, weakly negative and neutral weakly positive and strongly positive) classification tasks. The results obtained are compared favorably with those achieved by other systems addressing similar evaluations. [1]

2. Raymond Hsu, Bozhi See, Alan Wu in this paper "Machine Learning for Opinion Analysis on the Experience Project" proposed the following model

\section{a) Bag of Words (Bow)}

The Bow model is the most basic feature model in opinion analysis. It treats each unique word token as a separate feature. We use Bow features as our initial feature set for our system. This basic model acted as a test bench for us to observe the changes needed to make to our model better.

\section{b) WorldNet Synsets}

In order to improve the quality of the feature set and decrease overfitting, we used Word Net to map the words in the confessions onto their synonym set. By mapping words into their Synsets, we made the assumption that the words of similar meaning elicit similar emotions. This reduces the number of unique features we have and also improves the coverage of each feature. This technique also allows us to handle words that do not occur in our training data if they happen to be in the same Synsets as words that do occur in our training data.

\section{c) Opinion Lexicons}

Opinion lexicons are groupings of words into emotion and content categories. We used two of them in our system because we found they improved performance. We used them by replacing the original words with their opinion lexicon category. The first opinion lexicon we used was the Language Inquiry and Word Count (LIWC) (Penne baker et al., 2007), a hand engineered set of words and categories used by psychologists to group words by similar emotional and subject content. We also used features from the Harvard Inquirer (Stone et al., 1966), which also categorizes words by emotional and subject content. Like LIWC, the Harvard Inquirer was also hand-engineered by psychologists for the purpose of analyzing text. Both 
lexicons have been used in previous work on opinion analysis. [2]

3. Kuat Yessenov in his paper "Opinion Analysis of Movie Review Comments" presents two main approaches for classification: supervised and unsupervised. In supervised classification, the classifier is trained on labeled examples that are similar to the test examples. Contrary, unsupervised learning techniques assign labels based only on internal differences (distances) between the data points .Each sentence is considered independent from other sentences in classification approach. The labels we are interested in this project are (1) subjectivity of the sentence and (2) polarity of the sentence.

The author considers three supervised - Maximum Entropy, Naive Bayes, and Decision Trees, and one unsupervised classification approach - K-Means clustering. All four algorithms are available in NLTK framework.

Naive Bayes assumes that all features in the feature vector are independent, and Bayes' rule applies to the sentence. It calculates the prior probability frequency for each label in the training set and provides a likelihood estimate from the contributions of all features, and the label is assigned to the sentence with the highest likelihood estimate.

Maximum Entropy classifiers compute parameters that maximize the likelihood of the training corpus. The generalization of Naive Bayes classifiers is represented. The classifier applies iterative optimizations that find a local maximum. And the start state is initialized randomly. They are run multiple times during the training to find the best set of parameters.

Decision trees create a flowchart based classifier. It utilizes decision stumps at each level, simple classifiers that check for the presence of a single feature. The label is assigned to the sentence at the leaf nodes of the tree.

K-Means tries to find the natural clusters in the data, by calculating the distance from the centers of the clusters. The position of the centers is changed until the distances between all the points are minimal and centers are initially randomly assigned. K-Means can find only local maximum, and the final label assignment will be suboptimal. The common practice is to repeat the algorithm on the same data multiple times, and to represent the best result.

The author has analyzed the opinion of social network comments. He used the comments on articles from Digg as their text corpora. He evaluated the fitness of different feature selection and learning algorithms (supervised and unsupervised) on the classification of comments according to their subjectivity (subjective/objective) and their polarity (positive/ negative). The results show that simple bag-of-words model can perform relatively well, and it can be further refined by the choice of features based on syntactic and semantic information from the text. [3]

4. Minqing $\mathrm{Hu}$ and Bing Liu in this paper "Mining and summarizing customer reviews" proposed to build a lexicon of words with a known opinion for opinion classification. Opinion extraction techniques are very similar they are based on the adjectives surrounding the feature term. And the term differs from each other in the lexicon building process suggested using Word Net to build bipolar clusters. Starting with a head for each cluster, e.g. Fast for positive and slow for negative, subsets of these words be added to the corresponding clusters. It is based on the assumption that words that share the same orientation are synonyms and those having opposite orientations are antonyms. By having an initial seed, the lexicon can be expanded by following the Synsets in WorldNet. [4]

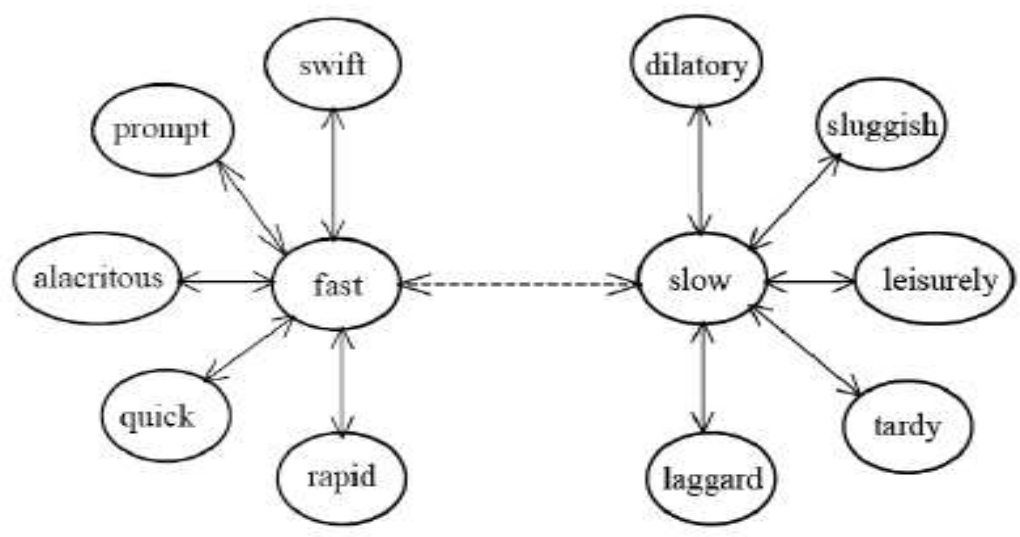

Fig. 1: Sample Bipolar Cluster suggested

\section{Proposed Work}

The basic idea of the proposed system is to develop natural language based feedback analysis system for feedback data which is capable of evaluating the feedback and provides result in appropriate form. The work mainly focuses on semantic mapping using the Jaccard similarity procedure followed by opinion 
analysis performed on the intermediate data for finding the polarity of the entity mentioned in the feedback data. Proposed architecture for this research work is as shown in figure 4.1. The architecture consists of seven phases which are explained in detail.

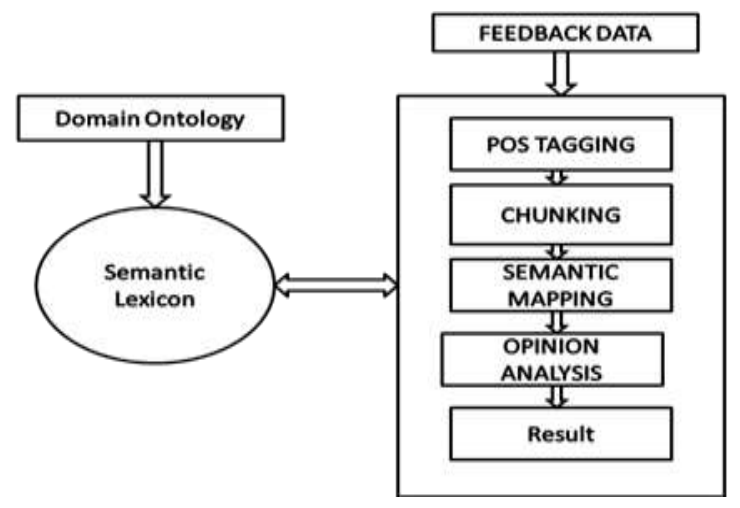

Fig. 2: Architecture of feedback analysis system

\subsection{Feedback Data Collection}

To create feedback database, feedbacks are collected from students with the help of feedback form. Students can express their views in free form about college, canteen, library, lab facility, extracurricular activities and teaching. In this way the feedback data is collected from the students of various departments of the institute. The input data collected from the students will help in analyzing and generating the result for the institute.

Example:

Financial support should be given to all.

Air conditioner should be installed.

Company Package is very less.

Trainer should be available for sports activities.

Space in Canteen is very limited.

Playground is not available.

\subsection{Pos Tagging}

The feedback data collected in the above step are analyzed and all the sentences are tagged with the help of the bi-directional dependency network tagger.

In the feedback analysis system the pos tagging is important for finding the pos tagged words from the sentences. The Stanford tagger is considered for the system. It is trained with $10,00,000$ words from the Oxford dictionary with the help of standard Maxnet tagger methods.

Part-of-speech tagging is often a critical first step in various speech and language processing tasks. Highaccuracy taggers (e.g., based on conditional random fields) rely on well chosen feature functions to ensure that important characteristics of the empirical training distribution are reflected in the trained model. This makes them vulnerable to any discrepancy between training and tagging corpora, and accuracy is adversely affected by the presence of out-of-vocabulary words. [6]

In corpus linguistics, word category disambiguation is the process of marking up the words in a text as corresponding to a particular part of speech it is based on its definition as well as context. It finds out the relationship with adjacent and related words in a phrase, sentence or paragraph. There are different pos tagged methods available like Stanford tagger, Latent Analogy, POSLDA, OPINE and based on their accuracy on Penn Treebank bank analysis the Stanford tagger was selected for the system that give an accuracy of $97.24 \%$.

The Stanford tagger uses the following ideas (i) explicit use of both preceding and following tag contexts via a dependency network representation, (ii) it uses a broad view of lexical features, on multiple consecutive words including jointly conditioning, (iii) in conditional log linear model priors are effectively used, and (iv) Fine-grained modeling of unknown word features. Bidirectional dependency network tagger in bidirectional/wsj3t0-18 holder gives $97.24 \%$ accuracy on the Penn Treebank WSJ, an error reduction of $4.4 \%$ of the best previous single automatically learned tagging result. The tagger uses a bi-directional dependency network tagger for tagging the words the tagger is composed of both the features of tagging; it uses a CMM method of left to right and right to left for extracting the tagged tokens.

Pre-processing of the sentence is done with the help of Stanford tagger. The Stanford tagger is included in the library of the program then the English bidirectional distsim tagger model is initialized form the library. The Maxnet tagger method is initialized to load the model file. The sentences are read sequentially with the help of a buffer reader $r$, then tokenize is used that reads data from the input and tokenizes it and return a list of word objects which can be fed into tag sentences. There are 36 tags available in Penn tree bank. Using these tags a list of tagged words is created and it is stored in an array of tagged list. Tagged sentences are printed with the tags information. The code below shows the step by step working of the Stanford bidirectional tagger used in the pre-processing of the sentences.

Examples:

Financial/JJ support/NN should/MD be/VB given/VBN to/TO all/DT. /.

Air/NN conditioner/NN should/MD be/VB installed/VBN./.

Company/NN Package/NN is/VBZ very/RB less/JJR./.

\subsection{Designing of Domain Ontology}

Ontology means defining the organization flow from top to bottom. The ontology designed the organization structure in the form of a binary tree diagram that helps 
in easily understanding the organization. There are certain steps that are necessary to follow in constructing the ontology model

The steps are as follows

1. Determine areas scope of domain ontology.

2. Examine the possibility of using existing ontology.

3. List important terms in the ontology.

4. Define classes and class hierarchy.

5. Define class attributes.

6. The definition of property distribution.

7. Create instances.

In the evaluation of the feedback system the institute is considered as the organization. Researchers have shown that it's hard to describe institute feedback concepts and their relationships using only one institute

Ontology model, it's possible to construct sub domain ontology models in organization sector. The mostly used ontology construction methods including Skeleton method, TOVE ontology, G\&FOX method, KACTUS And Bernaras methods, SENSUS, IDEF5 and seven-step method. Ontolingua, Ontodaurus, WebOnto, Protégé, OntoEdit are the common tools to construct ontology models.

The institute is considered as the basic and foremost ontology it comprises of all the entity that is related to the institute and cover almost all of the small and big entities within it.

The various sub domain entities like infrastructure, canteen, library, lab facility, extracurricular activities, teaching, and placement is further designed based on the properties so that it will be easy for classifying the entity.

The sub domain ontology's are designed following the necessary steps and considering the properties for each entity.

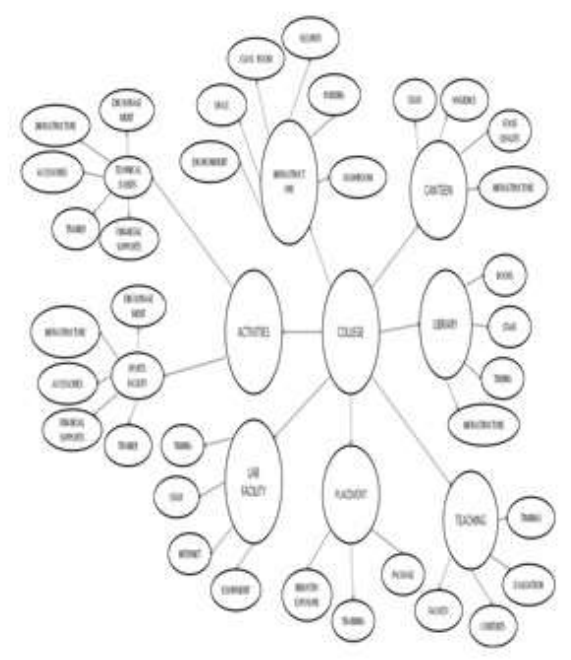

Fig. 3: Domain and Sub-Domain ontology

\subsection{Chunking}

Chunking is basically the identification of parts of speech and short phrases (like noun phrases). Part of speech tagging lets you are known whether words are nouns, verbs, adjectives, etc. The chunking takes place by combining all the terms that are specified in the grammar. The grammar is important in chunking that determines which words to chunk in same and different group.

In the feedback system chunking is used to chunk all the noun words from the sentences. A regular expression grammar is used that defines the NP words from the sentences, also known as a bag of words. These NP words or phrases will be used to match the words from the semantic lexicon and in order to determine which NP is related to which entity of the organization based on the properties. From the pos tagged words we have analyzed and extracted all the NP words with the help of grammar that defines the entity of the organization.

For example:

Sports/ NNS facility /NN are /VBP not /RB available IJJ. /.

\section{Chunked NP word}

(NP Sports/NNS facility/NN)

In the example all the noun words are chunked as a single NP word.

\subsection{Semantic Lexicon}

A lexicon is a list of words in a language-a vocabulary-along with some knowledge of how each word is used. It may be general or domain-specific for example, suppose a lexicon of several thousand common words of English or different languages. The words that are of use are usually open-class or content words, such as nouns, adjectives and verbs rather than closed-class or grammatical function words, such as articles, pronouns and prepositions, whose behavior is tightly bound to the grammar of the language. A lexicon may include multi-word expressions such as fixed phrases (by and large), phrasal verbs (tear apart), and other common expressions.

Each word or phrase in a lexicon is described in a lexical entry; exactly what is included in each entry depends on the purpose of the particular lexicon. The details that are given may include any of its properties of grammatical behavior, spelling or sound, meaning, or use, and the nature of its relationships with other words. A lexical entry is therefore a potentially large record specifying many aspects of the linguistic behavior and the meaning of a word.

In the feedback analysis system the semantic lexicon is created based on the input training data. The semantic lexicon includes the word phrases, the entity and its 
associated properties. The entities of the institute are studied in details and based on training data the word phrases are arranged so that they specific the entity more correctly all the possible combination for the entity are specified in the word phrases. The lexicon is designed in the table format and based on the training data the entries in the lexicon are increased.

Table 1: Semantic lexicon

\begin{tabular}{|c|c|c|}
\hline Words or phrases & Entity & Properties \\
\hline Contents & Teaching & Contents \\
\hline Samoa & Canteen & Items \\
\hline Books issue & Library & Books \\
\hline Trainer & Extracurricular activities & Sports activities \\
\hline Financial supports & Extracurricular activities & Sports activities \\
\hline Drinking & Canteen & Items \\
\hline Space & Canteen & Infrastructure \\
\hline
\end{tabular}

Like this the semantic lexicon is built for all the entities. The chunked words from the sentence are matched with the first column on the bases of the properties with the help of similarity matching method and if the matching is above the set threshold value the words or phrases are marked with the entity form the second column and are taken for further processing.

\subsection{Similarity Matching}

Measures of semantic similarity between concepts are widely used in Natural Language Processing. Similarity evaluation between two documents is an important operation which lies at the heart of most text and language processing tasks. The similarity evaluation forms a main part of the information retrieval system for retrieving the information.

There are different methods available for similarity matching. In our system we are not able to match the exact similarity between the post tagged words and the semantic lexicon because there are various human spelling errors that have to consider while evaluating similarity. Avoiding the human spelling error problem can become a great bottle neck for our system because it is not possible that all students will spell the words properly as described in the lexicon and by considering the exact similarity match it will not be possible for the system to match that word to the lexicon and all that word will not be considered resulting in degrading the performance of the feedback evaluation system.

So to solve this problem in the system implementing some similar method that matches the words and provide some similarity coefficient value so that by setting up some threshold value it will be possible to consider all the matching entries for the further evaluation and like this the problem of human spelling error can be overcome. There are various similarity evaluation methods such as cosine similarity, Jaccard similarity. In our system we had implemented Jaccard similarity.

Jaccard similarity determines the Jaccard coefficient. The Jaccard similarity coefficient is a statistical measure of similarity between sample sets and it is defined as the cardinality of their intersection divided by the cardinality of their union.

Mathematically,

$$
\begin{aligned}
& J(A, B)=|A \cap B| /|A U B| \\
& E g: X=\{A, B, C, D, E\}, Y=\{B, C, D, E, F\} \\
& X \text { and Y are words. }
\end{aligned}
$$

\section{Jaccard similarity $=4 / 6=0.67$}

In our system first we have extracted the post tagged words from all the feedback sentences from that we have chunked the NP words from all the sentences based on the chunking grammar that was defined for our system. The similarity match between the chunked words and the semantic lexicon is then calculated. The first column in the semantic lexicon contains the predefined words for the entity. We have taken the chunked word and had matched that word with the first column of the lexicon and for each match we have got the Jaccard coefficient value. Word pair with similarity score more than 0.7 are considered as similar. Second column of semantic lexicon describes entity and entity corresponding to the similar word is used in further processing.

For example Chunked words are Drinking water, Food quality, security, teaching of these words, and lexicon first column words are water, food quality, security, teachers which have Jaccard coefficient more than 0.7 and corresponding entities are canteen, canteen, infrastructure, and teaching. We have matched that word to the specified entity from the semantic lexicon so the final result is in terms of related to the party. Drinking water is related to canteen, food quality related to canteen, security related to infrastructure and teaching faculty related to teaching. Like this all the NP chunked words are matched to their entity of the organization with the help of the Jaccard similarity method.

The result that we have got by the similarity matching are compared based on the different training set of the semantic lexicon.

\subsection{Opinion Analysis}

A new approach to the opinion analysis has been developed that uses the polarity words for each ontology. The positive ontology, negative ontology, more ontology and inversion ontology are initialized.

In ontology the word are kept that defines their ontology. In positive ontology all the words that are kept define the positive nature of the entity. The 
positive ontology defines the entity as positive if the words of that ontology are specified in the sentences.

The negative ontology is same as positive ontology but it defines the negative polarity of the entity. In the negative ontology we have kept all the words that define the negative nature of the entity. The negative ontology defines the entity as negative if the words of that ontology are specified in the sentences.

The more ontology defines the extra nature of positive and negative ontology. The more ontology contains the word that defines how that word is more positive or more negative. These define an extra polarity of an entity. The words like very, more, extremely etc are used. The more ontology defines the entity as more negative if that word come before the negative ontology words and more positive if that word come before the positive ontology words.

The inversion ontology defines the nature that the word is positive or negative if the inversion ontology word appears before the positive ontology word it makes the sense of that word as negative for example if the positive word 'good' is present in the sentence and the inversion word 'not' appears before the positive word i.e. 'Not good'. It makes the negative polarity same as with the negative words if the inversion word appears before the negative ontology word then it makes the sense of that word as positive. For example

If the negative word 'bad' is present in the sentence and the inversion word 'not' appears before the negative words then it sense the word as positive.

By using this ontology an algorithm has been proposed that will help in deciding the polarity of the sentences.

\section{$\underline{\text { Algorithm for positive words }}$}

If (sentence. Contains (positive_feedback_ontology $[P C]))$

$$
\begin{aligned}
& \text { If (sentence. Contains (more ontology [ici])) } \\
& \text { If (index } 3<\text { index } 1)
\end{aligned}
$$

Good_count++;

If (sentence. Contains (inversion ontology [ic]))

$$
\text { If (index } 2<\text { index } 1 \text { ) }
$$

If $($ inv_word $==$ true $\& \&$ more_word $==$ true $)$

$$
\begin{aligned}
& \text { bad_count }+=2 ; \\
& \text { Good_count--; } \\
& \text { Else if }(\text { inv_word }==\text { false }) \\
& \text { Good_count }++; \\
& \text { Else } \\
& \text { Bad_count }++;
\end{aligned}
$$

The algorithm works in the following manner for positive words
First a pointer PC is declared that search for the positive feedback ontology word in the sentence it searches for all the words in the sentence. If the positive feedback ontology word is found it stores it's index in index 1 then it check for the more ontology word the pointer ici is initialized it check the more ontology word in the sentence if that word appears it stores it's pointer in index 3 then we check that if index 3 pointer is less then index 1 pointer then we increment the good count by 1 now we check for the inversion ontology we initialized a pointer ic if it searches for all inversion ontology word in the sentences if that word appears in the sentences it stores its index in index 2 . If index 2 is less then index 1 then the inv word in true then it check that if both inv word =true and more word =true then it increment the bad count by 2 and decrement the good count by 1 and if only inv word =true then it increment the bad count by 1 . If both inv word and more word are false then it increment the good count by 1.like this we get a good count for the positive word and a double count for the more positive word and bad count if the positive word is followed by an inversion word.

Algorithm for negative words

If (sentence. Contains (negative_feedback_ontology $[N C]))$

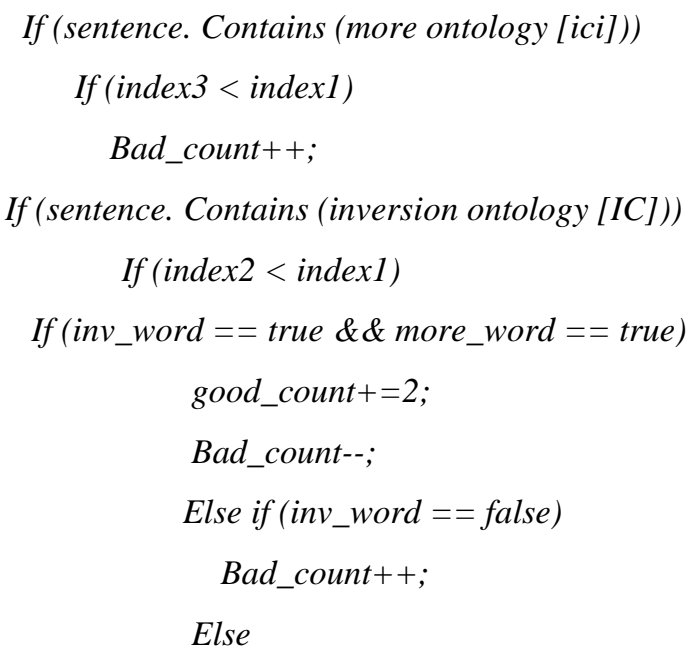

Good_count++;

The algorithm works in the following manner for negative words

First a pointer $\mathrm{NC}$ is declared that search for the negative feedback ontology word in the sentence it searches for all the words in the sentence. if the negative feedback ontology word is found it stores it's index in index 1 then it check for the more ontology word the pointer ici is initialized it check the more ontology word in the sentence if that word appears it stores it's pointer in index 3 then we check that if index 3 pointer is less then index 1 pointer then we increment the bad count by 1 now we check for the inversion ontology we initialized a pointer ic it searches for all inversion ontology word in the sentences if that word appears in the sentences it stores its index in index 2 . If 
index 2 is less then index 1 then the inv word in true then it check that if both inv word =true and more word $=$ true then it increment the good count by 2 and decrement the bad count by 1 and if only inv word =true then it increment the good count by 1 . If both inv word and more word are false then it increment the bad count by 1.like this we get a bad count for the negative word and a double count for the more negative word and good count if the negative word is followed by an inversion word.

In the feedback system these opinion method has been used and in the evaluation part the method is tested with the different lexicon and the test dataset.

\section{Experimental Result}

IR researchers have developed evaluation measures specifically designed to evaluate rankings. Most of these measures combine precision and recall in a way that takes account of the ranking. The results of the opinion analysis are computed by taking the parameters precision and recall.

Precision is one measure of the effectiveness of some computer applications for finding search words, candidate terms, and other items. Precision is a measure of the proportion of the results of a computer application that are considered to be pertinent or correct. For example, the system searches similarity match between the words and the semantic lexicon for the test data set and finds 304 sentences, 285 of which are really correct, then the system precession is $93.57 \%$.

Recall is one measure of the effectiveness of some computer applications for finding search words, candidate terms, and other items. Recall is a measure of the proportion of all possible correct results of a computer application that the application actually produces. For example, suppose you are using a computer application to search for terms in a document that has 80 terms in it. (You know because you counted them.) If the application finds 55 of these terms, then the recall of the application is 55 out of 80 , or 0.62 . The system searches similarity match between words and semantic lexicon that has 326 sentences in it. If the system finds 303 of these terms, then the recall of the system is 303 out of 326 , or $92.90 \%$.

In feedback analysis system these two parameters are evaluated for checking the accuracy of the system. In semantic analysis evaluation the three training dataset were considered of 500, 800, 1200 sentences. The semantic lexicon was trained with these sentences and precision and recall were calculated by varying this dataset. The test dataset contains 326 sentences these all sentences were tested on different semantic lexicon and result was calculated.

The table below shows all the data related to semantic analysis three datasets, their precision and recall value and the graph showing the variation in the result. [5]

Table2: Semantic analysis data

\begin{tabular}{|c|c|c|c|}
\hline SR NO & TRAINING DATA & $\begin{array}{c}\text { PRECISION } \\
(\%)\end{array}$ & $\begin{array}{c}\text { RECALL } \\
(\%)\end{array}$ \\
\hline 1 & 500 & 93.65 & 54.29 \\
\hline 2 & 800 & 99.65 & 88.34 \\
\hline 3 & 1200 & 99.67 & 92.94 \\
\hline
\end{tabular}

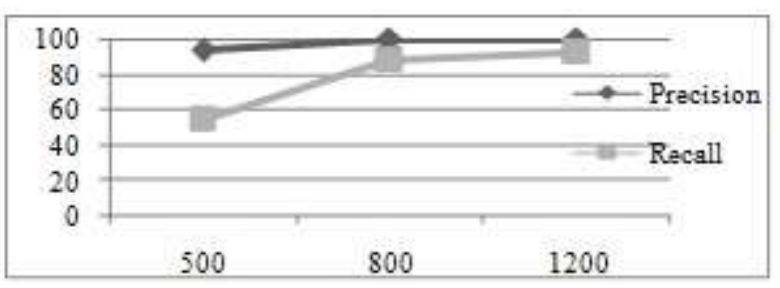

Fig. 4: Precision and Recall

From the result of the semantic analysis it is understood that the system will work more efficiently with the increase in size of the training data.

In opinion analysis evaluation, all the possible positive, negative, inversion and more words are added to the respective ontology's. The test dataset of 326 sentences evaluated on the feedback system and the result is calculated in the terms of precision.

The result contains all the entity of the institute and their precision value. The table below shows all the sub domain entity value the number of right and wrong value that have been calculated by the system for each entity based on these precision values a graph is plotted.

Table 3: Opinion analysis data

\begin{tabular}{|c|c|c|c|}
\hline ENTITY & $\begin{array}{c}\text { CORREC } \\
\text { TVALUE }\end{array}$ & $\begin{array}{c}\text { INCORRECT } \\
\text { VALUE }\end{array}$ & $\begin{array}{c}\text { PRECISION } \\
(\%)\end{array}$ \\
\hline Canteen & 43 & 11 & 79.62 \\
\hline Infrastructure & 34 & 12 & 73.91 \\
\hline Teaching & 29 & 5 & 85.24 \\
\hline Library & 32 & 9 & 78.04 \\
\hline Lab facility & 34 & 7 & 82.92 \\
\hline Placement & 45 & 7 & 86.53 \\
\hline Activities & 38 & 9 & 80.85 \\
\hline
\end{tabular}

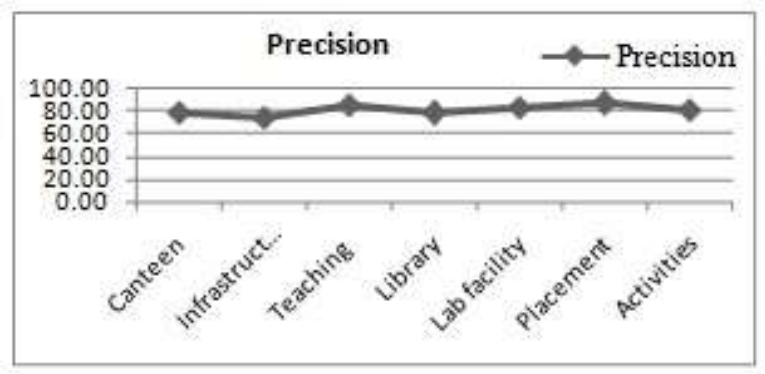

Fig. 5: Opinion Precision graph 


\section{Conclusion \& Future scope}

The natural language based feedback system will facilitated the students to freely express their views and suggestions for the institute that was not possible in existing feedback system. The use of natural language will benefited the system to a great extent. The Stanford tagger with accuracy of $97.63 \%$ are used to pos tagged the feedback sentences then the entity identification grammar enables the system to extract all the noun phrase from the sentences, semantic lexicon for the institute was created by observing the training datasets, semantic analysis helps in mapping the chunked NP words with their specified entity by the use of Jaccard similarity procedure and at last the opinion analysis was carried out by creating the opinion sets that helps in deciding the polarity of the sentences. Like this the feedback system was implemented. The precision and recall of the semantic analysis and opinion analysis provide the good result. The system is evaluated by the test dataset.

In Future work may continue in following directions:

- The system can be implemented for various domains.

- The system may also consider the audio feedback of the students.

- Designing the authoring tool.

\section{References}

[1] Jorge Carrillo de Albornoz, Laura Plaza, Pablo Gervás" A Hybrid Approach to Emotional Sentence Polarity and Intensity Classification" Proceedings of the Fourteenth Conference on Computational Natural Language Learning, pages 153-161, Uppsala, Sweden, 15-16 July 2010. C 2010 Association for Computational Linguistics.

[2] Raymond Hsu, Bozhi See, Alan Wu "Machine Learning for Opinion Analysis on the Experience Project" Computer Science Dept. Stanford Universityhsuray@cs.stanford.edu.BozhiSeeElectr icalEngineeringDept.StanfordUniversitybozhi@sta nford.eduAlanwuElectricalEngineeringDept.Stanfo rdUniversityalanw@stanford.edu published in 2011.

[3] Kuat Yessenov "Opinion Analysis of Movie Review Comments" 6.863 Spring 2009 final projects Kuat Yessenov kuat@csail.mit.edu Sa`sa Misailovi'c misailo@csail.mit.edu May 17, 2009.

[4] Minqing $\mathrm{Hu}$ and Bing Liu "Mining and summarizing customer reviews" department of computer science university of Illinois at Chicago 851 south Morgan street Chicago, IL 60607-7053 \{mhu1,liub\}@cs.uic.edu kdd'04, august 22-25, 2004, Seattle, Washington, USA.
[5] Pratik Agrawal, Prof. A. J. Agrawal "Implementation of Semantic Analysis Using Domain Ontology" (Department of Computer Science, Shri Ramdeobaba College of Engineering \& Management Nagpur, India IOSR Journal of Computer Engineering (IOSR-JCE) e-ISSN: 22780661, p- ISSN: 2278-8727Volume 11, Issue 2 (May. - Jun. 2013), PP 72-76.

[6] Kristina Toutanova, Dan Klein "Feature-Rich Partof-Speech Tagging with a Cyclic Dependency Network" Computer Science Stanford University Stanford, CA 94305-9040 kristina@cs.stanford.edu ,klein@cs.stanford.edu Manning (2011).

\section{Authors' Profiles}

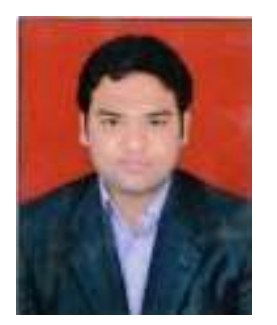

Pratik K. Agrawal: received Bachelor of Engineering Degree in Information Technology from Amravati University, and Master of Technology degree in Computer Science \& Engineering from Shri Ramdeobaba College of Engineering \& Management Nagpur, India in 2007 and 2013 respectively. His research area is Natural Language Processing. He is having 11 months of teaching experience. Presently he is Assistant Professor in Prof Ram Meghe Institute of technology \& Research, India $\mathrm{He}$ is the author of two research papers in International Journal, Conferences

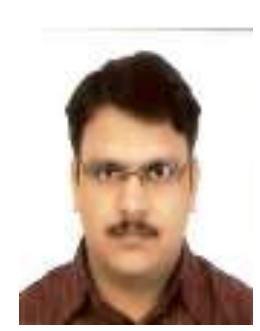

Avinash J. Agrawal: received Bachelor of Engineering Degree in Computer Technology from Nagpur University, India and Master of Technology degree in Computer Technology from National Institute of Technology, Raipur, India in 1998 and 2005 respectively. $\mathrm{He}$ received Ph.D. from Visvesvaraya National Institute of Technology, Nagpur, India in 2013. His research area is Natural Language Processing and Databases. He is having 15 years of teaching experience. Presently he is Assistant Professor in Shri Ramdeobaba College of Engineering \& Management Nagpur, India $\mathrm{He}$ is the author of seven research papers in International and National Journal, Conferences.

How to cite this paper: Pratik K. Agrawal, Avinash. J. Agrawal,"Opinion Analysis Using Domain Ontology for Implementing Natural Language Based Feedback System", IJITCS, vol.6, no.3, pp.61-69, 2014. DOI: $10.5815 /$ ijitcs.2014.03.08 